\title{
Influência da capacidade de campo na taxa de crescimento do cafeeiro conilon ${ }^{1}$
}

\author{
Maria Christina Junger Delôgo Dardengo², Edvaldo Fialho dos Reis ${ }^{3}$, Renato Ribeiro Passos ${ }^{4}$
}

\begin{abstract}
RESUMO
O objetivo deste trabalho foi avaliar a influência da capacidade de campo (CC) na taxa de crescimento do cafeeiro conilon a partir da adoção de três tensões: 0,006 MPa $\left(\mathrm{CC}_{1}\right), 0,010 \mathrm{MPa}\left(\mathrm{CC}_{2}\right)$ e $0,033 \mathrm{MPa}\left(\mathrm{CC}_{3}\right)$, em dois tipos de solo (Latossolo Vermelho-Amarelo e Argissolo Vermelho-Amarelo). O experimento foi montado em casa de vegetação no Núcleo de Estudos e de Difusão de Tecnologia em Floresta, Recursos Hídricos e Agricultura Sustentável, município de Jerônimo Monteiro, Espírito Santo. A espécie vegetal utilizada foi a Coffea canephora Pierre ex A. Froehner, variedade Robusta Tropical (EMCAPER 8151), cultivada em vaso de 12 litros, por um período de 255 dias. As análises de crescimento foram realizadas 15 dias após o transplantio das mudas e no final do experimento, para determinação de matéria seca total e área foliar. $\mathrm{O}$ teor de umidade do solo na capacidade de campo varia com a tensão adotada em sua determinação. As maiores taxas de crescimentos relativo e absoluto do cafeeiro conilon foram obtidas quando a umidade do solo foi mantida na capacidade de campos determinada na tensão de 0,010 MPa no Latossolo VermelhoAmarelo e de 0,006 MPa no Argissolo Vermelho-Amarelo. As menores taxas de crescimento da cultura foram observadas na capacidade de campo determinada na tensão de 0,033 MPa, o que inviabiliza a sua adoção na estimativa da lâmina de irrigação utilizando-se a câmara de pressão de Richards.
\end{abstract}

Palavras-chave: Café, crescimento vegetal, umidade do solo.

\section{ABSTRACT}

\section{Influence of field capacity on the growth rate of Conilon coffee}

The objective of this work was to evaluate the influence of field capacity (FC) on the growth rate of the conilon coffee plant by using three tensions ( $0.006 \mathrm{MPa}\left(\mathrm{FC}_{1}\right), 0.010 \mathrm{MPa}\left(\mathrm{FC}_{2}\right)$ and $0.033 \mathrm{MPa}\left(\mathrm{FC}_{3}\right)$ ) in two soils (Red-Yellow Oxisol and Red-Yellow Ultisol). The experiment was carried out in a greenhouse at the Center of Studies and Technology Dissemination in Forestry, Water Resources and Sustainable Agriculture, in the municipality of Jerônimo Monteiro-ES. Plants of Coffea canephora Pierre ex A. Froehner, variety Robusta Tropical (EMCAPER 8151), were cultivated in 12 L containers for 255 days. Growth analyses were carried out 15 days after transplanting and at the end of the experiment total dry matter and leaf area were determined. The soil moisture level in the field capacity varied as a function of the tension chosen for its determination. The highest rates of relative and absolute growth of Conilon coffee plants were obtained when the soil moisture was kept close to the field capacity determined at $0.010 \mathrm{MPa}$ in the Red-Yellow Oxisol and at $0.006 \mathrm{MPa}$ in the Red-Yellow Ultisol. The lowest growth rates of the crop were found in the field capacity determined at $0.033 \mathrm{MPa}$, making it unfeasible to the use in the estimation of the irrigation sheet with the Richard's pressure plate apparatus.

Key words: Coffee plant, plant growth, soil moisture.

\footnotetext{
Recebido para publicação em maio de 2008 e aprovado em dezembro de 2009

${ }^{1}$ Extraído da Dissertação apresentada pelo primeiro autor ao Programa de Pós-Graduação em Produção Vegetal do Centro de Ciências Agrárias (CCA) da Universidade Federal do Espírito Santo (UFES), Alegre, Espírito Santo.

${ }^{2}$ Engenheira-Agrônoma, Mestre. UFES, Campus de Alegre, 29530-000, Distrito de Rive, Alegre, Espírito Santo. mchrisjunger@hotmail.com

${ }^{3}$ Engenheiro-Agrícola, Doutor. Departamento de Engenharia Rural, CCA, UFES, 29530-000, Alegre, Espírito Santo. edreis@ cca.ufes.br

${ }^{4}$ Engenheiro-Agrônomo, Doutor. Departamento de Fitotecnia, CCA, UFES, 29530-000, Alegre, Espírito Santo. renatopassos@ cca.ufes.br
} 


\section{INTRODUÇÃO}

O crescimento do cafeeiro é influenciado por vários fatores, destacando-se os genéticos e edafoclimáticos. Além do acúmulo de matéria seca, pode-se também expressar o crescimento de uma espécie utilizando a matéria seca produzida num período de tempo, por meio da taxa de crescimento absoluto (TCA) e da taxa de crescimento relativo (TCR) (Bragança, 2005).

Com a crescente demanda da técnica de irrigação, torna-se necessário o uso racional da água, a qual deve ser aplicada em tempo oportuno e na quantidade correta. Para precisa quantificação do fornecimento de água às plantas é imprescindível conhecer a relação funcional entre umidade do solo e o seu potencial matricial na zona radicular das culturas, conhecida como curva de retenção de água no solo (Dourado Neto et al., 1990).

A determinação da curva de retenção ou curva característica de água do solo é de suma importância na área de irrigação e nos estudos de movimento de água no solo, pois fornece o teor de água do solo $(\theta)$ em diferentes tensões ( $\Psi)$, e vice-versa. Assim, propicia condições necessárias para a determinação do teor de água disponível no solo, do teor de água atual e de outras variáveis básicas à execução do manejo adequado da água de irrigação e à quantificação dos processos dinâmicos envolvendo o sistema solo-planta-atmosfera. A curva de retenção pode ser obtida pelo processo de secagem da amostra previamente saturada ou por umedecimento gradual da amostra de terra fina seca ao ar (TFSA). Entretanto, para o manejo da irrigação prefere-se o processo de secagem (Costa et al., 2008).

A umidade do solo é um dos fatores limitantes da produtividade agrícola. Assim, o solo constitui-se no principal reservatório de água para as plantas. Nos projetos de irrigação é considerada como água disponível às plantas aquela do intervalo de umidade entre a capacidade de campo e o ponto de murcha permanente. A capacidade de campo representa o máximo conteúdo de água retido pelo solo depois que o excesso tenha sido drenado e a taxa de movimento descendente tenha decrescido acentuadamente (Veihmeyer \& Hendrickson, 1931); ou seja, o limite superior desse intervalo. Já o limite inferior é representado pelo ponto de murcha permanente, que se refere ao teor de água no solo caracterizado pela forte retenção matricial, abaixo do qual a planta não consegue absorver água (Mello et al., 2002).

A capacidade de campo pode ser determinada por métodos de campo e de laboratório. O método clássico de laboratório foi descrito por Richards (1947), tendo sido denominado de câmara de pressão de Richards, que fixou o potencial matricial da capacidade de campo em -0,033 MPa. Entretanto, Reichardt (1988) afirmou que nos solos característicos das regiões tropicais e úmidas esse critério deve ser alterado para potenciais maiores na determinação da capacidade de campo, da ordem de -0,010 MPa e -0,006 MPa.

Desde então, muitas tentativas têm sido feitas em laboratório para associar a capacidade de campo com o conteúdo de água do solo em equilíbrio com 0,033 MPa e 0,010 MPa (Souza \& Reichardt, 1996; Van Lier, 2000). Bernardo et al. (2005) consideraram que a tensão correspondente à capacidade de campo para solos de textura fina é de 0,033 MPa e para solos de textura grossa de 0,010 MPa. Já Ruiz et al. (2003), ao pesquisarem 80 Latossolos e oito Neossolos Quartzarênicos, observaram que a capacidade de campo ocorre, nesses solos, a potenciais maiores que -0,033 MPa. Por sua vez, a tensão de 0,006 MPa foi sugerida por Andrade et al. (1991) e Ferreira \& Marcos (1983) como possível estimativa da umidade correspondente à capacidade de campo. Diante do exposto, questiona-se qual a correta tensão associada à capacidade de campo quando se utiliza o método de laboratório câmara de pressão de Richards, em cada tipo de solo, uma vez que, segundo Fabian \& Ottoni Filho (2000), ainda não há uma posição consensual entre os pesquisadores.

Levando-se em consideração esses aspectos, objetivou-se com este trabalho avaliar a influência da umidade na capacidade de campo (CC) determinada nas tensões de 0,006 $\mathrm{MPa}\left(\mathrm{CC}_{1}\right)$, 0,010 $\mathrm{MPa}\left(\mathrm{CC}_{2}\right)$ e 0,033 $\mathrm{MPa}$ $\left(\mathrm{CC}_{3}\right)$, na taxa de crescimento do cafeeiro conilon, em dois tipos de solos (Latossolo Vermelho-Amarelo e Argissolo Vermelho-Amarelo).

\section{MATERIAL E MÉTODOS}

O experimento foi conduzido em casa de vegetação no Núcleo de Estudos e de Difusão de Tecnologia em Floresta, Recursos Hídricos e Agricultura Sustentável (NEDTEC), município de Jerônimo Monteiro-ES, localizado nas coordenadas $20^{\circ} 47^{\prime} 25^{\prime \prime} \mathrm{S}$ e $41^{\circ} 23^{\prime} 48^{\prime \prime}$ W a $120 \mathrm{~m}$ de altitude, vinculado ao Centro de Ciências Agrárias da Universidade Federal do Espírito Santo (CCA-UFES).

Os solos utilizados foram o Latossolo Vermelho-Amarelo (LVA) e Argissolo Vermelho-Amarelo (PVA), coletados à profundidade de $0,00-0,30 \mathrm{~m}$. As amostras dos solos foram submetidas ao esboroamento e passagem em peneira de $2 \mathrm{~mm}$, destinadas a análises física e química. A análise granulométrica apresentou os seguintes resultados analíticos: LVA - areia: 316,0 g. $\mathrm{kg}^{-1}$; silte: 105,0 g. $\mathrm{kg}^{-1}$; argila: 579,0 g. $\mathrm{kg}^{-1}$; e PVA - areia: 532,0 g.kg-1; silte: 256,0 g. $\mathrm{kg}^{-1}$; e argila: 212,0 g. $\mathrm{kg}^{-1}$. Pela análise química verificouse: LVA- 0,3; 2,1; $1,3 \mathrm{cmol}_{\mathrm{c}} \mathrm{dm}^{-3} \mathrm{de} \mathrm{K}^{+}, \mathrm{Ca}^{2+} \mathrm{e} \mathrm{Mg}^{2+}$, respectivamente; $\mathrm{pH}$ 5,4; $19 \mathrm{~g} \mathrm{~kg}^{-1}$ de $\mathrm{MO}$ e 6,0 $\mathrm{mg} \mathrm{dm}^{-3}$ de P; e PVA - 0,$1 ; 1,8 ; 0,8 \mathrm{cmol}_{\mathrm{c}} \mathrm{dm}^{-3} \mathrm{de} \mathrm{K}^{+}, \mathrm{Ca}^{2+} \mathrm{e} \mathrm{Mg}^{2+}$, respectivamente; $\mathrm{pH}$ 5,6; $18 \mathrm{~g} \mathrm{~kg}^{-1}$ de $\mathrm{MO}$ e 3,0 $\mathrm{mg} \mathrm{dm}^{-3}$ de $\mathrm{P}$. 
Foram realizadas as adubações corretiva e nutricional, de acordo com o Manual de Calagem e Adubação para o Estado do Espírito Santo - 5a aproximação (Prezotti et al., 2007).

As curvas de retenção de água do Latossolo Vermelho-Amarelo e Argissolo Vermelho-Amarelo foram determinadas por meio de extratores de placas de cerâmica, por secamento, conforme EMBRAPA (1997), adotando-se um tempo mínimo não inferior a três dias nas tensões de 0,006 ; 0,010; 0,033; 0,08; 0,10; 0,30; 0,50; 1,0; e 1,5 MPa, com três repetições. Os valores médios de umidade volumétrica da curva de retenção de cada solo foram ajustados utilizando-se o modelo matemático proposto por Van Genuchten (1980), descrito na equação 1:

$$
\theta=\theta \mathrm{r}+(\theta \mathrm{s}-\theta \mathrm{r}) /\left[1+(\alpha . h)^{\mathrm{n}}\right]^{\mathrm{m}}
$$

em que:

$\theta$ = umidade à base de volume;

$\theta \mathrm{r}=$ umidade volumétrica residual a $15000 \mathrm{~cm}$ de água;

$\theta \mathrm{s}=$ umidade volumétrica de saturação;

$\mathrm{h}=$ tensão em cm de água; $\mathrm{e}$

$\alpha, \mathrm{n}$ e $\mathrm{m}=$ parâmetros empíricos determinados pelo software Soil Water Retention Curves (SWRC, versão 2.00), elaborado por Dourado Neto et al. (2000).

A água disponível (AD) foi calculada a partir dos valores de umidade volumétrica observados na curva de retenção para a capacidade de campo (CC) determinada na tensão de 0,006 MPa (CC $)$, 0,010 MPa $\left(\mathrm{CC}_{2}\right)$ e 0,033 $\mathrm{MPa}\left(\mathrm{CC}_{3}\right)$ e ponto de murcha permanente (PMP) na tensão de 1,5 MPa, utilizando-se a seguinte expressão: $\mathrm{AD}=$ CC - PMP (Centurion \& Andrioli, 2000). A lâmina de irrigação (L) foi calculada pela expressão: $\mathrm{L}=\mathrm{AD} \times \mathrm{V}_{\mathrm{s}}$, sendo $\mathrm{AD}$ água disponível e $\mathrm{V}_{\mathrm{S}}$ volume de solo do vaso (0,012 $\left.\mathrm{m}^{3}\right)$. Para transformar a lâmina de irrigação $(\mathrm{L})$ em volume (mL/vaso), multiplicou-se L pela área do vaso $\left(0,048 \mathrm{~m}^{2}\right)$. O estabelecimento e o controle de umidade na $\mathrm{CC}_{1}, \mathrm{CC}_{2} \mathrm{e}$ $\mathrm{CC}_{3}$ foram feitos pelo monitoramento do peso do vaso da repetição $1\left(R_{1}\right)$ de cada tratamento, incluindo solo, planta e umidade. As irrigações foram realizadas manualmente por diferença de pesagem, sendo a umidade do solo elevada até a capacidade de campo. $\mathrm{O}$ ajuste dos pesos dos vasos a umidade gravimétrica $\left(\mathrm{UG}_{\mathrm{E}}\right)$ para os níveis da capacidade de campo foi feito mensalmente, devido ao incremento propiciado pelo crescimento das plantas.

As análises de crescimento foram realizadas 15 dias após o transplantio das mudas nos vasos e no final do experimento (255 dias). Para obtenção da matéria seca total $\left(\mathrm{MS}_{\mathrm{T}}\right)$, as plantas foram secas em estufa a $75^{\circ} \mathrm{C}$ até atingirem peso seco constante. A área foliar foi medida a partir de um integrador de área foliar, modelo LI-3100, da marca Li-cor. Com base nesses dados, foram determina- das a taxa de assimilação líquida $\left[\mathrm{TAL}=\left[\left(\mathrm{P}_{2}-\mathrm{P}_{1}\right) /\left(\mathrm{t}_{2}-\mathrm{t}_{1}\right)\right]\right.$ $\mathrm{x}\left[\left(\operatorname{Ln} \mathrm{A}_{2}-\operatorname{Ln} \mathrm{A}_{1}\right) /\left(\mathrm{A}_{2-} \mathrm{A}_{1}\right)\right]$, a taxa de crescimento relativo $\left[T C R=\left(\operatorname{Ln} \mathrm{P}_{2}-\operatorname{Ln} \mathrm{P}_{1}\right) /\left(\mathrm{t}_{2}-\mathrm{t}_{1}\right)\right]$ e a taxa de crescimento absoluto $\left[T C A=\left(\mathrm{P}_{2}-\mathrm{P}_{1}\right) /\left(\mathrm{t}_{2}-\mathrm{t}_{1}\right)\right]$. P representa a massa seca total (parte aérea e raiz), $\mathrm{t}_{1}$ o tempo na primeira coleta, $\mathrm{t}_{2}$ o tempo na última coleta e A representa a área foliar (Benincasa, 2003).

\section{RESULTADOS E DISCUSSÃO}

A Tabela 1 apresenta as equações do modelo matemático proposto por Van Genuchten (1980), cujos parâmetros empíricos ( $\alpha$, n e m) foram obtidos conforme Dourado Neto et al. (2000), destinadas ao ajuste das curvas de retenção de água do Latossolo VermelhoAmarelo (LVA) e Argissolo Vermelho-Amarelo (PVA) na profundidade de $0,00-0,30 \mathrm{~m}$.

Os desvios entre a umidade volumétrica observada e ajustada para cada tensão da curva de retenção são apresentados na Tabela 2. Nota-se que para baixas tensões (0,0001 a 0,10 MPa) o método de laboratório câmara de pressão de Richards superestimou o conteúdo de água dos solos, com exceção da tensão de 0,010 MPa, e para altas tensões ( 0,30 a $1,5 \mathrm{MPa})$ subestimou, resultando em acréscimos e decréscimos em seus valores nos distintos pontos da curva. Resultado semelhante foi obtido por Costa et al. (2008), que testaram diferentes modelos de ajuste e métodos para a determinação da curva de retenção de água de um LVA e constaram que as maiores diferenças foram observadas nos teores de água ajustados na faixa de teor de água mais elevado. Observaram ainda que o modelo de Van Genuchten torna-se mais confiável em comparação a outros modelos utilizados no ajuste da curva de retenção de água no solo, por apresentar coeficiente angular mais próximo de 1 e intercepto mais próximo de zero.

Pela Figura 1, nota-se que as curvas de retenção do LVA e PVA não são semelhantes e que os maiores conteúdos de água estão relacionados às tensões mais baixas, ou seja, valores mais altos de potencial matricial. Observa-se que os conteúdos de água do LVA, de textura argilosa, foram mais elevados do que os do PVA, de textura franco-argilo-arenosa, em todas as tensões do intervalo disponível, realçando a importância da fração argila na retenção de água pelo solo. Isso está de acordo com Reichardt (1996), que considera a textura o principal

Tabela 1. Equações de ajustes para o Latossolo VermelhoAmarelo (LVA) e Argissolo Vermelho-Amarelo (PVA) segundo modelo de Van Genuchten (1980) ${ }^{(1)}$

\begin{tabular}{lc}
\hline Solo & ${ }^{(1)} \theta=\theta r+(\theta s-\theta r) /\left[1+(\alpha . h)^{\mathrm{n}}\right]^{\mathrm{m}}$ \\
LVA & $\theta=0,239+(0,453-0,239) /\left[1+(0,026859 . h)^{1,527469}\right]^{0,345322}$ \\
PVA & $\theta=0,129+(0,488-0,129) /\left[1+(0,097296 . h)^{1,502570}\right]^{0,334474}$ \\
\hline
\end{tabular}


Tabela 2. Umidade volumétrica observada $\left(\theta_{\mathrm{O}}\right)$ e umidade volumétrica ajustada $\left(\theta_{\mathrm{A}}\right)$ pela equação de Van Genuchten (1980) para o Latossolo Vermelho-Amarelo (LVA) e Argissolo Vermelho-Amarelo (PVA)

\begin{tabular}{|c|c|c|c|c|c|c|}
\hline \multirow{2}{*}{ Tensão (MPa) } & LVA_ $\theta_{0}$ & $\mathbf{L V} \_\theta_{\mathrm{A}}$ & \multirow{2}{*}{ Desvio } & PVA_ $\theta_{\theta}$ & PVA_ $\theta_{A}$ & \multirow{2}{*}{ Desvio } \\
\hline & \multicolumn{2}{|c|}{$\mathbf{m}^{3} \mathbf{m}^{-3}$} & & \multicolumn{2}{|c|}{$\mathbf{m}^{3} \mathbf{m}^{-3}$} & \\
\hline 0,0001 & 0,453 & 0,453 & 0,000 & 0,488 & 0,484 & $-0,004$ \\
\hline 0,006 & 0,388 & 0,384 & $-0,004$ & 0,281 & 0,274 & $-0,007$ \\
\hline 0,010 & 0,342 & 0,358 & 0,016 & 0,229 & 0,242 & 0,013 \\
\hline 0,033 & 0,317 & 0,306 & $-0,011$ & 0,204 & 0,192 & $-0,012$ \\
\hline 0,08 & 0,287 & 0,281 & $-0,006$ & 0,175 & 0,169 & $-0,006$ \\
\hline 0,10 & 0,283 & 0,277 & $-0,006$ & 0,170 & 0,165 & $-0,005$ \\
\hline 0,30 & 0,265 & 0,260 & $-0,005$ & 0,151 & 0,150 & $-0,001$ \\
\hline 0,50 & 0,251 & 0,255 & 0,005 & 0,143 & 0,145 & 0,003 \\
\hline 1,0 & 0,240 & 0,250 & 0,010 & 0,132 & 0,140 & 0,008 \\
\hline 1,5 & 0,239 & 0,248 & 0,009 & 0,129 & 0,138 & 0,009 \\
\hline
\end{tabular}

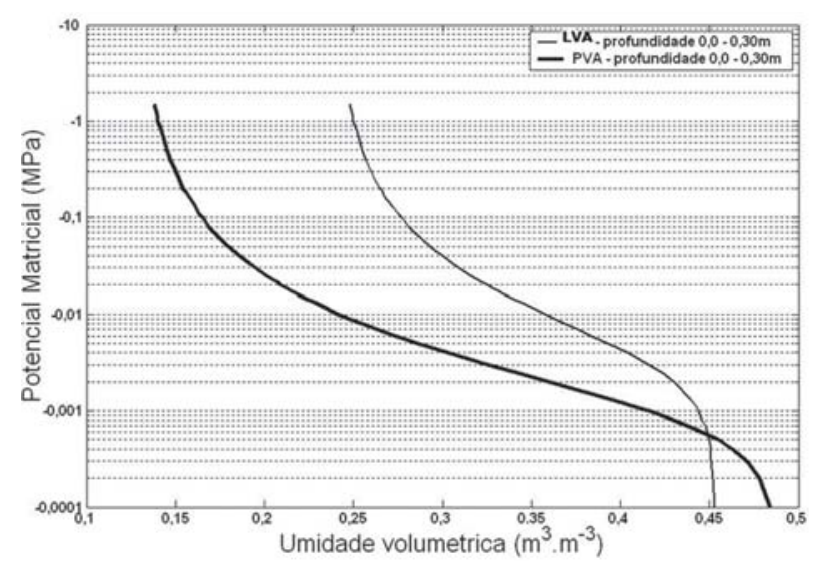

Figura 1. Curvas de retenção de água do Latossolo VermelhoAmarelo (LVA) e Argissolo Vermelho-Amarelo (PVA) à profundidade de $0,00-0,30 \mathrm{~m}$.

determinante da retenção de água e, de maneira geral, que solos de textura fina, com elevadas proporções de silte e argila, possuem maior capacidade de armazenamento de água.

Os valores de umidade volumétrica e conteúdos de água disponível nos níveis de capacidade de campo $\left(\mathrm{CC}_{1}\right.$, $\mathrm{CC}_{2}$ e $\mathrm{CC}_{3}$ ) do LV e PVA são apresentados na Figura 2. Verifica-se que o teor de umidade na capacidade de campo varia com a tensão adotada em sua determinação, utilizando-se a câmara de pressão de Richards. Assim, a umidade volumétrica obtida nas tensões de 0,$006 ; 0,010$; e 0,033 MPa foi de 38,4; 35,8; e 30,6\% para o LVA e de 27,4; 24,2; e 19,2\% para o PVA, respectivamente. Com isso, foram estabelecidos valores distintos de água disponível para o mesmo solo. Os conteúdos de água disponível nas tensões de 0,010 MPa e 0,033 MPa em relação aos obtidos na tensão de 0,006 MPa representam 80,88 e 42,64\% no LVA e 76,47 e 39,71\% no PVA, respectivamente. Todavia, percebe-se que os menores valores de água disponível foram obtidos na capacidade de campo determinada na tensão de 0,033 MPa em ambos os solos estudados.
Desse modo, depreende-se que a capacidade de campo determinada na tensão de 0,033 MPa não representa a máxima retenção de água do LVA e PVA, o que é corroborado por Reichardt (1988), uma vez que a retenção de água nessa tensão está associada à baixa atividade das argilas e textura dos solos. Isso irá influenciar no manejo da irrigação, uma vez que está diretamente relacionado com as necessidades hídricas das culturas, características hidráulicas do sistema de irrigação selecionado e com a capacidade de retenção de água pelo solo na profundidade efetiva da raiz de uma cultura específica (Soares et al., 1998).

Os resultados da análise de crescimento do cafeeiro conilon, a partir da determinação da taxa de crescimento absoluto (TCA), taxa de crescimento relativo (TCR) e taxa assimilatória livre (TAL), são apresentados na Tabela 3. A TAL representa o incremento de matéria seca por unidade de material assimilatório (área foliar) por tempo, resultante dos efeitos dos tratamentos. A importância da área foliar é amplamente conhecida por ser um parâmetro indicativo de produtividade, pois o processo fotossintético depende da interceptação da energia luminosa da sua conversão em energia química. Assim sendo, a superfície foliar é a base do rendimento potencial da cultura (Favarin et al., 2002). Por sua vez, a taxa de crescimento relativo (TCR) é um índice que representa a dinâmica de acúmulo de matéria seca, ao longo do tempo, relacionado à matéria seca inicial, sendo dependente da taxa assimilatória livre. Já a taxa de crescimento absoluto (TCA) representa a matéria seca produzida por unidade de área ou planta durante certo tempo (Bragança, 2005).

As variações observadas no crescimento da cultura podem ser explicadas pela taxa assimilatória livre (TAL), que expressa o desempenho do sistema assimilatório das plantas em função dos tratamentos a que foram submetidas. A maior eficiência da superfície foliar associada à maior área foliar culminará na maior taxa de crescimentos relativo e absoluto da cultura, conforme pode ser obser- 

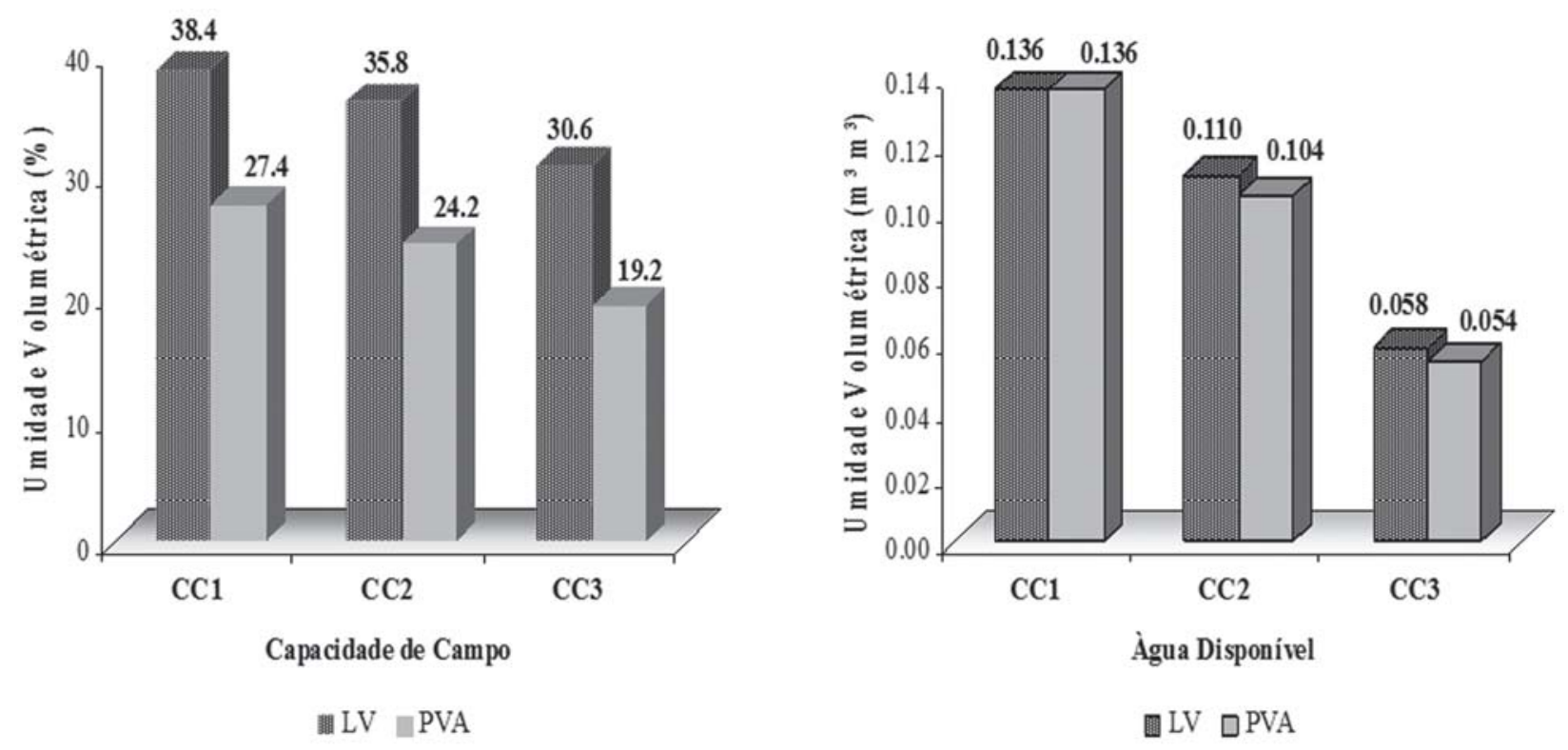

Figura 2. Umidade volumétrica e conteúdos de água disponível nos níveis de capacidade de campo $\left(\mathrm{CC}_{1}=0,006 \mathrm{MPa} ; \mathrm{CC}_{2}=0,010\right.$ MPa e $\mathrm{CC}_{3}=0,033 \mathrm{MPa}$ ) do Latossolo Vermelho-Amarelo (LVA) e Argissolo Vermelho-Amarelo (PVA).

Tabela 3. Taxa assimilatória livre (TAL), taxa de crescimento relativo (TCR) e taxa de crescimento absoluto (TCA) do cafeeiro conilon nos níveis de capacidade de campo $\left(\mathrm{CC}_{1}=0,006 \mathrm{MPa} ; \mathrm{CC}_{2}=0,010 \mathrm{MPa}\right.$ e $\left.\mathrm{CC}_{3}=0,033 \mathrm{MPa}\right)$ do Latossolo VermelhoAmarelo (LVA) e Argissolo Vermelho-Amarelo (PVA)

\begin{tabular}{lcccc}
\hline Solo & Capacidade de campo & TAL $\left(\mathbf{g ~ c m}^{-\mathbf{2}} \mathbf{d i a}^{-\mathbf{1}}\right)$ & TCR(g g $\left.\mathbf{~ d i a}^{-\mathbf{1}}\right)$ & TCA(g dia \\
\hline LVA & $\left.\mathrm{CC}_{1}\right)$ & 0,0179 & 0,5418 \\
& $\mathrm{CC}_{2}$ & $2,9217^{-04}$ & 0,0184 & 0,6154 \\
& $\mathrm{CC}_{3}$ & $3,2731^{-04}$ & 0,0152 & 0,2909 \\
PVA & $2,3689^{-04}$ & 0,0147 & 0,3296 \\
& $\mathrm{CC}_{1}$ & $2,3748^{-04}$ & 0,0125 & 0,1967 \\
& $\mathrm{CC}_{2}$ & $1,9959^{-04}$ & 0,0103 & 0,1157 \\
\hline
\end{tabular}

vado na Tabela 3. Nota-se que houve diferença no crescimento das plantas em relação ao tipo de solo e aos níveis de capacidade de campo. Os valores de TCR, TCA e TAL obtidos no LVA foram mais elevados do que no PVA, considerando-se o mesmo nível de capacidade de campo. A taxa de crescimento absoluto (TCA) do cafeeiro conilon na capacidade de campo determinada nas tensões de 0,006 $\mathrm{MPa}\left(\mathrm{CC}_{1}\right)$ e $0,033 \mathrm{MPa}\left(\mathrm{CC}_{3}\right)$ corresponde, respectivamente, a 88,04 e 47,27\% da maior TCA obtida na tensão de 0,010 MPa no LVA $\left(\mathrm{CC}_{2=}\right.$ 0,6154 $\left.\mathrm{g} \mathrm{dia}^{-1}\right)$. Da mesma forma, a TCA na capacidade de campo determinada nas tensões de $0,010 \mathrm{MPa}\left(\mathrm{CC}_{2}\right)$ e $0,033 \mathrm{MPa}\left(\mathrm{CC}_{3}\right)$ corresponde, respectivamente, a 59,68 e 35,10\% da maior TCA obtida na tensão de 0,006 MPa do PVA $\left(\mathrm{CC}_{1}=0,3296 \mathrm{~g} \mathrm{dia}^{-1}\right)$.

Com isso, observa-se que a umidade da capacidade de campo determinada na tensão de 0,010 MPa promoveu a maior taxa de crescimento absoluto do cafeeiro conilon no Latossolo Vermelho-Amarelo, superando em 53,55\% a maior taxa de crescimento absoluto obtida na tensão de 0,006 MPa no Argissolo Vermelho-Amarelo. Contudo, quando a tensão de 0,033 MPa foi utilizada na determina- ção da capacidade de campo $\left(\mathrm{CC}_{3}\right)$, foram obtidas as menores taxas de crescimento do cafeeiro conilon, o que inviabiliza a sua adoção na estimativa da lâmina de irrigação em ambos os solos estudados.

\section{CONCLUSÕES}

O teor de umidade na capacidade de campo varia com a tensão adotada em sua determinação utilizando-se a câmara de pressão de Richards, o que implica no cálculo da água disponível do solo e manejo da irrigação.

A capacidade de campo determinada na tensão de 0,010 MPa para o Latossolo Vermelho-Amarelo e de 0,006 MPa para o Argissolo Vermelho-Amarelo promove as maiores taxas de crescimento do cafeeiro conilon.

As menores taxas de crescimento da cultura são observadas quando a tensão de 0,033 MPa é utilizada na determinação da capacidade de campo do Latossolo Vermelho-Amarelo e Argissolo Vermelho-Amarelo, o que inviabiliza a sua adoção na estimativa da lâmina de irrigação utilizando-se a câmara de pressão de Richards. 


\section{REFERÊNCIAS}

Andrade CLT, Freitas JAD \& Luz LRQP (1991) Características físico-hídricas de solos arenosos de tabuleiros litorâneos. In: $9^{\circ}$ Congresso Nacional de Irrigação e Drenagem, Natal. Anais, ABIS. p.1069-1095.

Benincasa MMP (2003) Análise de crescimento de plantas, noções básicas, 2 ed. Jaboticabal, FUNEP. 41p.

Bernardo S, Soares AA \& Mantovani EC (2005) Manual de irrigação, 7 ed. Viçosa, UFV- Imprensa Universitária. 611p.

Bragança SM (2005) Crescimento e acúmulo de nutrientes pelo cafeeiro conilon (Coffea canephora Pierre). Tese de Doutorado. Universidade Federal de Viçosa, Viçosa, 99p.

Centurion JF \& Andrioli I (2000) Regime hídrico de alguns solos de Jaboticabal. Revista Brasileira da Ciência do Solo, 24:701709

Costa WA, Oliveira CAS \& Kato E (2008) Modelos de ajuste e métodos para a determinação da curva de retenção de água de um Latossolo Vermelho-Amarelo. Revista Brasileira da Ciência do Solo, 32:515-523.

Dourado Neto D, Nielsen DR, Hopmans JW, Reichardt K \& Bacchi OOS (2000) Software to model soil water retention curves (SWRC, version 2.00). Scientia Agricola, 57:191-192.

Dourado Neto D, Van Lier QJ, Botrel TA \& Libardi PL (1990) Programa para confecção da curva de retenção de água no solo utilizando o modelo de Genuchten. Engenharia Rural, 1:92102 .

EMBRAPA - Empresa Brasileira de Pesquisa Agropecuária (1997) Manual de métodos de análise de solos, 2 ed. Rio de Janeiro, MAA. 212p.

Fabian AJ \& Ottoni Filho TB (2000) Determinação da capacidade de campo in situ ou através de equações de regressão. Pesquisa Agropecuária Brasileira, 35:1029-1036.

Favarin JL, Dourado Neto D, Garcia AG, Villa Nova NA \& Favarin MGGV (2002) Equações para estimativa do índice foliar do cafeeiro. Pesquisa Agropecuária Brasileira, 37:769-773.
Ferreira MM \& Marcos ZZ (1983) Estimativa da capacidade de campo de Latossolo Roxo distrófico e Regossolo através do ponto de inflexão da curva característica de umidade. Ciência e Prática, 7:96-101.

Mello CR, Oliveira GC, Resck JML \& Junior MSD (2002) Estimativa da capacidade de campo baseada no ponto de inflexão da curva característica. Ciência Agrotécnica, 26:836-841.

Prezotti LC, Gomes JA, Dadalto GG, Oliveira JA, de (2007) Manual de recomendação de calagem e adubação para o Estado do Espírito Santo - 5a aproximação. Vitória, SEEEA/INCAPER/ CEDAGRO. 305p.

Reichardt K (1996) Dinâmica da matéria e da energia em ecossistemas. Piracicaba, ESALQ/USP. 513p.

Reichardt K (1988) Capacidade de campo. Revista Brasileira da Ciência do Solo, 12:211-216.

Richards LA (1947) Pressure-membrane apparatus, construction and use. Agronomy Engineering, 28:451-454.

Ruiz HA, Ferreira GB \& Pereira JBM (2003) Estimativa da capacidade de campo de Latossolos e Neossolos Quartzarênicos pela determinação do equivalente de umidade. Revista Brasileira da Ciência do Solo, 27:389-393.

Soares JM, Costa FF \& Santos CR (1998) Manejo de irrigação em fruteiras. In: Faria MA, Silva EL, Vilela L, Vilela LAA \& Silva AM (Eds.). Manejo de irrigação. Poços de Caldas, Sociedade Brasileira de Engenharia Agrícola. p.281-310.

Souza DL \& Reichardt K (1996) Estimativas da capacidade de campo. Revista Brasileira da Ciência do Solo, 20:183-189.

Van Genuchten MT (1980) A closed-form equation for predicting the hydraulic conductivity of unsaturated soils. Soil Science Society of American Journal, 41:892-898.

Van Lier QJ (2000) Índice da disponibilidade de água para as plantas. In: Novais RF, Alvarez VH \& Schaefer CEGR. Tópicos em ciência do solo. Viçosa, Sociedade Brasileira de Ciência do Solo. p.95-106.

Veihmeyer FJ \& Hendrickson AH (1931) The moisture equivalent as a measure of the field capacity of soils. Soil Science, 32:181-193. 